\title{
Multi-targeted kinase inhibition alleviates mTOR inhibitor resistance in triple-negative breast cancer
}

\author{
Jichao He ${ }^{1} \cdot$ Ronan P. McLaughlin ${ }^{1}$. Vera van der Noord ${ }^{1}$. John A. Foekens ${ }^{2}$. John W. M. Martens ${ }^{2}$. \\ Gerard van Westen ${ }^{1} \cdot$ Yinghui Zhang $^{1} \cdot$ Bob van de Water $^{1}[$
}

Received: 21 February 2019 / Accepted: 27 July 2019 / Published online: 6 August 2019

(c) The Author(s) 2019

\begin{abstract}
Purpose Owing to its genetic heterogeneity and acquired resistance, triple-negative breast cancer (TNBC) is not responsive to single-targeted therapy, causing disproportional cancer-related death worldwide. Combined targeted therapy strategies to block interactive oncogenic signaling networks are being explored for effective treatment of the refractory TNBC subtype. Methods A broad kinase inhibitor screen was applied to profile the proliferative responses of TNBC cells, revealing resistance of TNBC cells to inhibition of the mammalian target of rapamycin (mTOR). A systematic drug combination screen was subsequently performed to identify that AEE788, an inhibitor targeting multiple receptor tyrosine kinases (RTKs) EGFR/ HER2 and VEGFR, synergizes with selective mTOR inhibitor rapamycin as well as its analogs (rapalogs) temsirolimus and everolimus to inhibit TNBC cell proliferation.

Results The combination treatment with AEE788 and rapalog effectively inhibits phosphorylation of mTOR and 4EBP1, relieves mTOR inhibition-mediated upregulation of cyclin D1, and maintains suppression of AKT and ERK signaling, thereby sensitizing TNBC cells to the rapalogs. siRNA validation of cheminformatics-based predicted AEE788 targets has further revealed the mTOR interactive RPS6K members (RPS6KA3, RPS6KA6, RPS6KB1, and RPS6KL1) as synthetic lethal targets for rapalog combination treatment.

Conclusions mTOR signaling is highly activated in TNBC tumors. As single rapalog treatment is insufficient to block mTOR signaling in rapalog-resistant TNBC cells, our results thus provide a potential multi-kinase inhibitor combinatorial strategy to overcome mTOR-targeted therapy resistance in TNBC cells.
\end{abstract}

Keywords Multi-kinase inhibitor · mTOR-targeted therapy $\cdot$ Drug resistance $\cdot$ Triple-negative breast cancer (TNBC) . Polypharmacology

\section{Abbreviations \\ BL Basal-like \\ CDK4 Cyclin-dependent kinase 4 \\ CI Combination index \\ EGF Human epidermal growth factor}

Electronic supplementary material The online version of this article (https://doi.org/10.1007/s10549-019-05380-z) contains supplementary material, which is available to authorized users.

Bob van de Water

b.water@lacdr.leidenuniv.nl

1 Division of Drug Discovery and Safety, Leiden Academic Centre for Drug Research, Leiden University, 2300 RA Leiden, The Netherlands

2 Department of Medical Oncology and Cancer Genomic Netherlands, Erasmus MC Cancer Institute, Erasmus Medical Centre, 3000 CA Rotterdam, The Netherlands
FC Fold change

IC50 Half-maximal inhibitory concentration

IM Immunomodulatory

KI Kinase inhibitor

LAR Luminal androgen receptor-like

MSL Mesenchymal stem-like

mTOR The mammalian target of rapamycin

RTK Receptor tyrosine kinase

SRB Sulforhodamine B

TNBC Triple-negative breast cancer

\section{Background}

Triple-negative breast cancer (TNBC) constitutes a small subtype (10-20\%) of breast cancer, but causes the majority of breast cancer-related deaths $[1,2]$. As defined by the 
absence of ER and PR expression and HER2 overexpression, TNBC is not curable by hormone receptor or HER2-targeted therapies [3]. Furthermore, TNBC is highly heterogeneous. Gene expression profiling has further classified TNBC into six unique molecular subtypes, namely basal-like (BL1 and BL2), mesenchymal (M), mesenchymal stem-like (MSL), immunomodulatory (IM), and luminal androgen receptorlike (LAR) subtype [4]. The TNBC molecular signatures have been explored for targeted therapies in clinical trials, including those targeting receptor tyrosine kinases (RTKs, e.g., EGFR, VEGFR, c-Met), PI3K/AKT, Ras/MAPK, JAK/ STAT, cell cycle regulators $[5,6]$. Yet, TNBC has not benefited from above mono-targeted therapies so far, due to intrinsic or acquired resistance [6].

The mammalian target of rapamycin (mTOR), a conserved serine/threonine protein kinase, is a central regulator of cell growth and proliferation, by sensing and integrating multiple signals from growth factors and nutrient signals [7, 8]. mTOR hyperactivity is frequently observed in TNBC compared to other breast cancer subtypes and is often correlated with poor prognosis, underpinning the potential of mTOR-targeted therapy for TNBC treatment [9-11]. Although mTOR-targeted interventions, such as rapamycin and its analogs (rapalogs) temsirolimus and everolimus, delay progression and extend survival, patients with TNBC eventually develop resistance to mTOR inhibitors with undesired outcome [9, 12]. Evidence has shown that rapalog treatment could release mTOR negative feedback on upstream kinases and activate compensatory pathways, for instance, PI3K/AKT and MAPK/ERK signaling pathways, thereby bypassing mTOR inhibition [13-15]. This observation underscores the need for alternative combinatorial therapeutic approaches for TNBC treatment.

Since oncogenic pathways incorporate multiple signaling components and axes to promote tumor malignancy, monotherapy may not be sufficient for long-term control of TNBC $[9,13,16]$. Hence, simultaneously targeting different signaling molecules represents a promising strategy to impede tumor growth and progression $[8,17]$. Several reports have documented that co-targeting growth factor receptors and mTOR exerts cooperative anti-cancer effects in various cancer types, including TNBC [18-22]. However, these studies focus on a particular combination in the questioned cancer type. Little is known about the interactive kinases involved in rapalog resistance and the mechanisms of the combinatorial effect remain unclear. Here, we systematically screened a broad collection of kinase inhibitors across a large panel of TNBC lines treated with rapamycin. Our data demonstrated that multiple targeted kinase inhibition, for instance, by inhibitor AEE788, sensitizes TNBC cells to various mTOR inhibitors, rapamycin, temsirolimus, and everolimus. Integrated cheminformatics study and siRNA validation revealed additional putative targets of AEE788, which interact closely with mTOR signaling. Most importantly, our study provided an efficacious approach for exploring cancer combination treatment. Moreover, the combinatorial therapy is more effective than single drug application and thus demonstrates a therapeutic advantage over either agents as a monotherapy in TNBC treatment.

\section{Methods}

\section{Kinase inhibitor library combination screen}

One day post seeding into 96-well plates, cells were treated with individual kinase inhibitors alone or combined with rapamycin at $1 \mu \mathrm{M}$. After 4-day treatment, proliferation was evaluated by sulforhodamine B (SRB) colorimetric assay [23]. Detailed information on materials and methods can be found in Supplementary file ESM_3.

\section{Results}

\section{TNBC cell lines are differentially responsive to $\mathrm{mTOR}$ inhibitor rapalogs}

To gain insights into TNBC dependency on mTOR signaling integration for proliferation and cell survival, a KI library $\left(\right.$ Selleckchem ${ }^{\circledR}$ ) containing 378 small molecular inhibitors targeting various kinase signaling pathways was screened across 19 TNBC cell lines (Suppl. Table S1), which are representative for the six transcriptome-based subtypes of TNBC [4]. All TNBC cell lines were exposed to individual inhibitors at $1 \mu \mathrm{M}$ for 4 days, followed by measurement of cell proliferation. The effect of each inhibitor on proliferation was assessed by $Z$ scores normalized to overall proliferative response. TNBC cell lines were largely resistant to the majority of the kinase inhibitors, without any clear correlation to the TNBC molecular subtypes (Fig. 1a). The proliferative response towards mTOR inhibitors was variable among TNBC cell lines. We distinguished 11 TNBC cell lines insensitive to different mTOR inhibitors (Fig. 1b), including rapamycin (Rap) and its analogs (i.e., rapalogs), zotarolimus, everolimus, ridaforolimus, and temsirolimus. HCC1806 and SUM149PT were most resistant to rapologs, while Hs578T was most sensitive.

Rapalogs are highly selective allosteric inhibitors of mTOR, by binding to FKBP12/rapamycin-binding domain to block mTOR Ser2448 phosphorylation and function [24, 25]. mTOR Ser2448 is a predominant phosphorylation residue for mTOR kinase activity in response to mitogenderived stimuli [25]. Therefore, we examined the inhibitory effect of rapamycin (Rap), temsirolimus (Tem), and everolimus (Eve), on Ser2448-mTOR phosphorylation with 


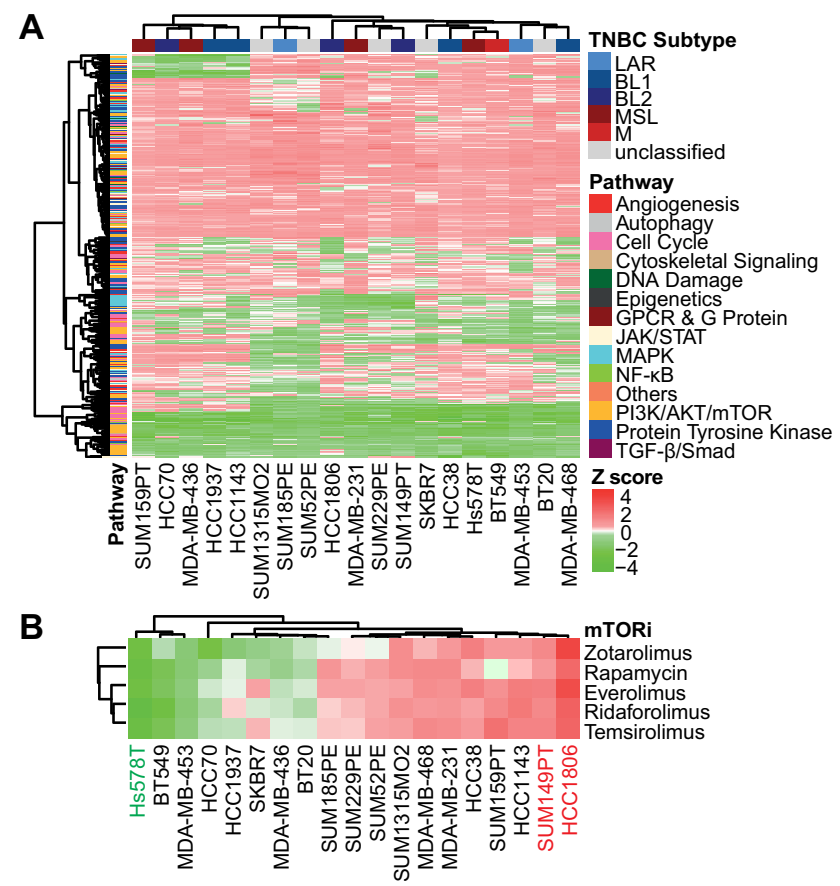

Fig. 1 Resistance profiling of TNBC cell lines to mTOR inhibitor rapalogs. a Heatmap presenting the responses of 19 TNBC cell lines to 378 kinase inhibitors. Data were shown based on the effect of individual $\mathrm{KI}$ on proliferation (relative $Z$ scores), subtype-annotated cell lines (clustered horizontally), and pathway-annotated inhibitors (clustered vertically). Strong inhibitory effect on proliferation was indicated in green and weak in red. b Response clustering of TNBC cell lines to mTOR inhibitors (mTORi). c Concentration range effects

a focus on rapalog-resistant TNBC cell lines HCC1806 and SUM149PT and rapalog-sensitive Hs578T TNBC cells. The rapalogs potently inhibited phosphorylation of mTOR in the sensitive Hs578T cells, but not or less effectively in the resistant HCC1806 and SUM149PT cells, respectively (Fig. 1c, d). These data suggest that mTOR kinase activity and its sustained phosphorylation render the TNBC cells resistant to rapalogs.

\section{Combinatorial drug screen identifies kinase inhibitors sensitizing TNBC cells to mTOR inhibition}

Next, to identify kinase inhibitors synergizing with mTOR inhibition in rapalog refractory TNBC cells, we further performed a drug screen with rapamycin (at $1 \mu \mathrm{M})$ in combination with the 378 kinase inhibitors (also tested at $1 \mu \mathrm{M})$ in the resistant SUM149PT cells. Pearson's correlation coefficient $r$ displayed high reproducibility of two replicate screens for $\mathrm{KI}(r=0.9509)$ and $\mathrm{KI}$ and rapamycin (KI+Rap, $r=0.9115$ ), respectively (Fig. 2a, b). Comparison of KI+Rap combinatory effect to the single KI effect on proliferation inhibition uncovered 9 potent KIs (Fig. 2c), which significantly enhanced inhibitory effect of rapamycin
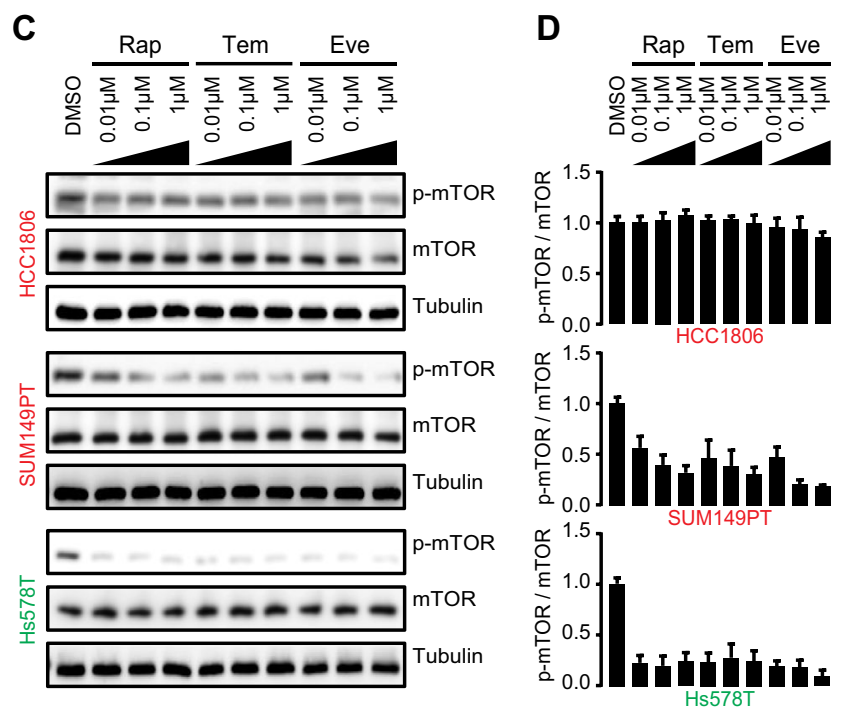

of rapalogs rapamycin (Rap), temsirolimus (Tem), and everolimus (Eve) on mTOR phosphorylation, in rapalog-resistant HCC1806 and, SUM149PT TNBC cells, compared to rapalog-sensitive Hs578T cells. Cells were treated with rapalogs in concentration range $(\mu \mathrm{M})$ for $4 \mathrm{~h}$. d Quantitative comparison of phosphorylated mTOR level to total mTOR level in rapalog-treated resistant and sensitive TNBC cells

on proliferation of SUM149PT cells (Fig. 2d). These included one MEK inhibitor PD184352 and 8 RTK inhibitors, AEE788, afatinib, AC480, AZD8931, AZD9291, AST1306, ZM 306416, and gefitinib that are described to target single or multiple EGFR/HER2 and VEGFR RTKs (Fig. 2e). We also performed rapamycin combination screen in the resistant HCC1806 cells in parallel. As HCC1806 cells were responsive to EGFR inhibitors, only additive effects were observed (Suppl. Fig. S1c; Suppl. Fig. S2).

These data implicate that while the resistant SUM149PT cells poorly respond to inhibitors of EGFR or VEGFR and mTOR inhibitor rapamycin alone, concurrent blockage of upstream EGFR or VEGFR RTK activity or MEK signaling transduction, and downstream mTOR signaling could converge re-sensitization of TNBC cells.

\section{Multi-targeted RTK inhibitor AEE788 enhances proliferative inhibition and cell death in rapalog-resistant TNBC cells}

Next, we further validated the combinatorial effect of the most promising combinations on proliferative inhibition in the rapalog-resistant SUM149PT cells. We focused on the 
A

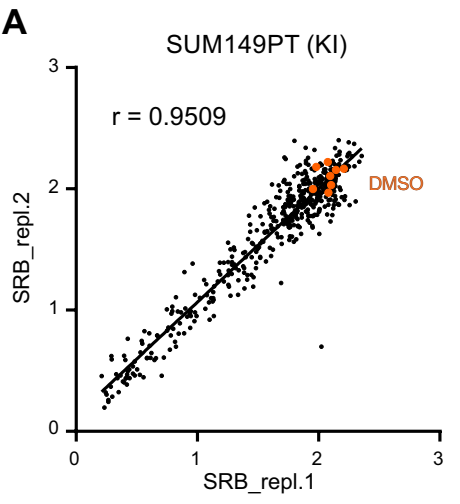

D

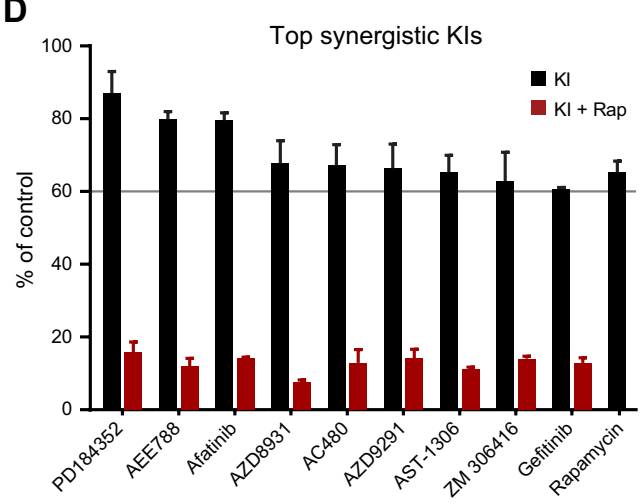

B

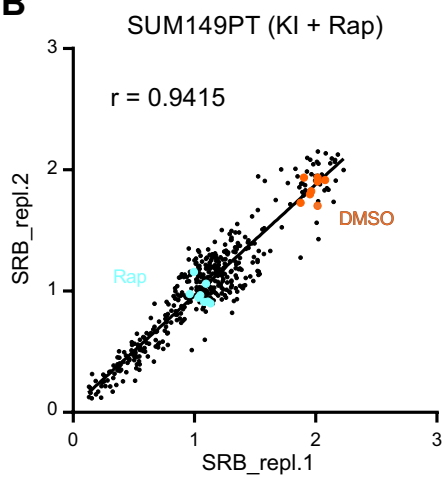

C

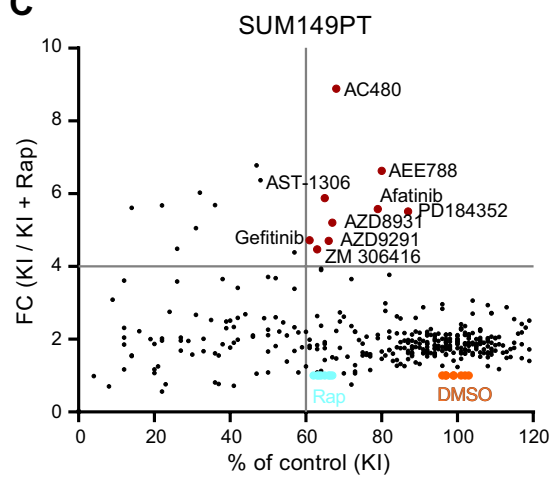

E

\begin{tabular}{|cc|}
\hline KI & Targets (IC50/nM) \\
\hline PD184352 & MEK1/2 (17) \\
AEE788 & EGFR (2), HER2 (6), VEGFR1 (59) \\
Afatinib & EGFR (0.5), HER4 (1) \\
AC480 & EGFR (20), HER2 (30) \\
AZD8931 & HER2 (3), EGFR (4) \\
AZD9291 & EGFR (494) \\
AST-1306 & EGFR (0.5), HER4 (0.8) \\
ZM 306416 & VEGFR1 (330), SRC (330) \\
Gefitinib & EGFR Tyr1173 (37) \\
\hline
\end{tabular}

Fig. 2 Identification of kinase inhibitors which sensitizes TNBC cells to rapamycin. a, b Pearson's correlation coefficient $r$ showing reproducibility of replica screen of 378 kinase inhibitors alone (KI, a) or combined with rapamycin (KI+ Rap, b). SUM149PT cells were treated for 4 days with $1 \mu \mathrm{M}$ KI individuals alone or combined with $1 \mu \mathrm{M}$ Rap. Orange dots, DMSO control. Cyan dots, Rap only. $\mathbf{c}$ Effect comparison of KI alone to KI combined with Rap on proliferation of SUM149PT cells. The percentage of proliferation (\% of control) was

MEK inhibitor PD184352, EGFR inhibitor gefitinib, and the multi-targeted RTK inhibitor AEE788. Cells were treated with rapamycin in a concentration range alone or combined with different concentrations of PD184352, gefitinib, or AEE788. AEE788 synergized with rapamycin to inhibit SUM149PT cell proliferation in dose-dependent manner (Fig. 3a, top panel). In contrast, PD184352 and gefitinib (Suppl. Fig. S1a, b) displayed a more additive effect when combined with rapamycin. The synergistic effects of AEE788 and rapalogs, Rap, Tem, and Eve, were further confirmed in SUM149PT as well as another rapalog-resistant TNBC cell line HCC1143 (Fig. 3a). AEE788 significantly reduced the half-maximal inhibitory concentrations (IC50) of the rapalogs in both SUM149PT and HCC1143 cell lines (Fig. 3b). Combination index (CI) analysis detected the strong synergy of AEE788 and rapalogs, overall with CI values $<0.5$ (Fig. $3 \mathrm{c}$ ). To detect the combinatorial effects of AEE788 and rapamycin on TNBC cell death, we performed Annexin V/Propidium Iodide apoptosis assay in SUM149PT and HCC1143 cell lines. Besides proliferative inhibition, the combination significantly enhanced

relative to DMSO. The ratio of percentage of proliferation was shown as fold change (FC, KI versus $\mathrm{KI}+\mathrm{Rap}$ ). Top synergistic inhibitors were marked in red. d Selected inhibitors reducing $40 \%$ proliferation with FC $>4$ (extracted from $\mathbf{c}$, red dots) when combined with rapamycin. Error bars indicate screen replicates. e Kinase targets of the selected inhibitors and IC50 values of the inhibitors on corresponding targets $\left(\right.$ SelleckChem ${ }^{\circledR}$ )

apoptosis and necrosis $96 \mathrm{~h}$ post treatment in SUM149PT cells (Fig. 3d), and enhanced the monotherapy-induced apoptosis in HCC1143 cells, albeit not statistically significant (Fig. 3e). Next, we evaluated the combinatorial effects on normal mammary cells MCF10A and renal cells RPTEC. Importantly, neither monotherapy nor combination significantly suppressed proliferation or induced cell death of MCF10A and RPTEC cells, suggesting that the combo effects might be cancer cell specific and less toxic in normal mammary and renal cells (Suppl. Fig. S3). Altogether, the KI combination not only inhibited TNBC cell proliferation, but also enhanced the monotherapy-induced apoptosis and necrosis, with minimal effects on normal mammary and renal cells. 
A
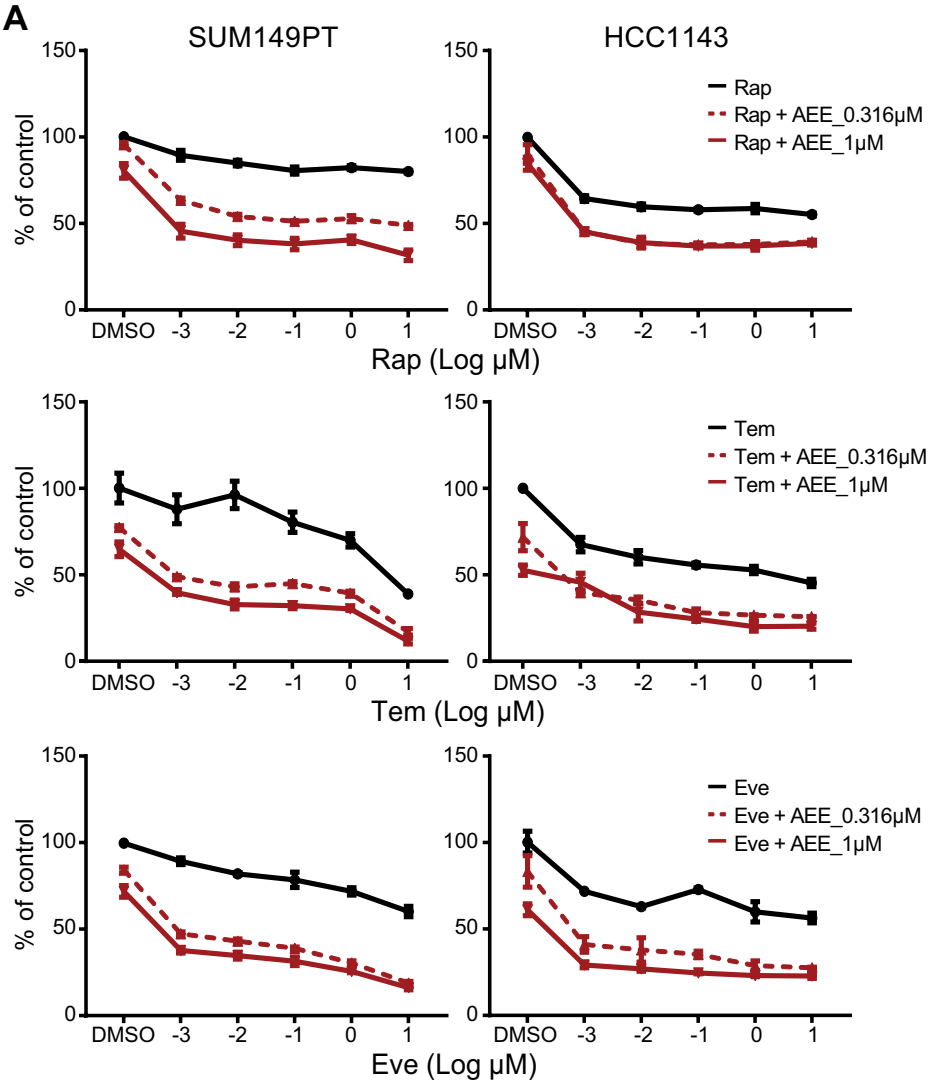

D

SUM149PT
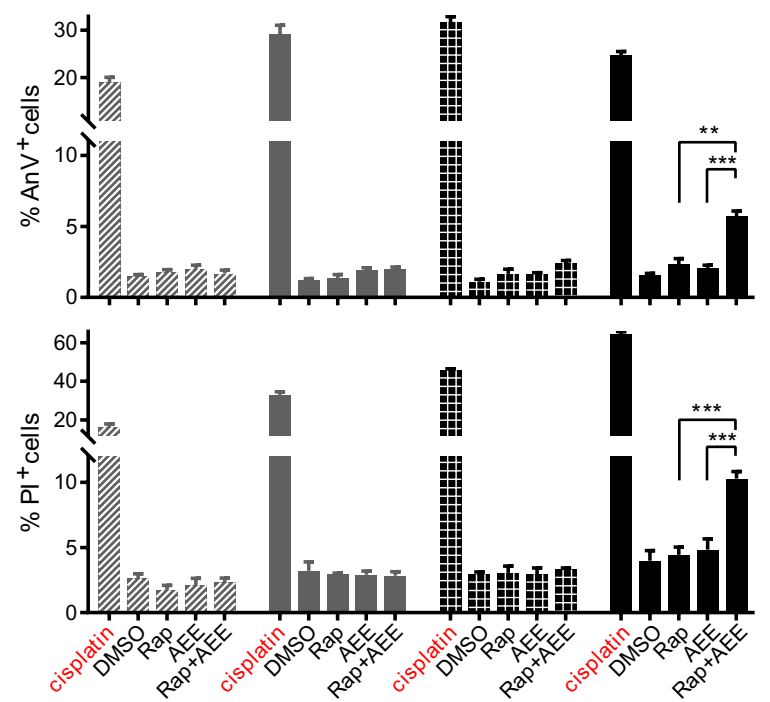

Fig. 3 Synergistic effect of AEE788 and rapalogs on proliferative inhibition and cell death in rapalog-resistant TNBC cells. a Proliferative response of rapalog-resistant SUM149PT (left panel) and HCC1143 (right panel) TNBC cells, to rapalogs Rap, Tem, and Eve in concentration range alone or combined with $0.316 \mu \mathrm{M}$ and $1 \mu \mathrm{M}$ AEE788, respectively. b IC50 values $(\mu \mathrm{M})$ of rapalogs in combination with AEE788, inducing $50 \%$ of proliferation inhibition in SUM149PT and HCC1143 cells. c Combination index (CI)
B

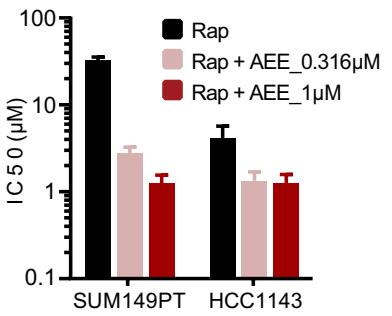

C
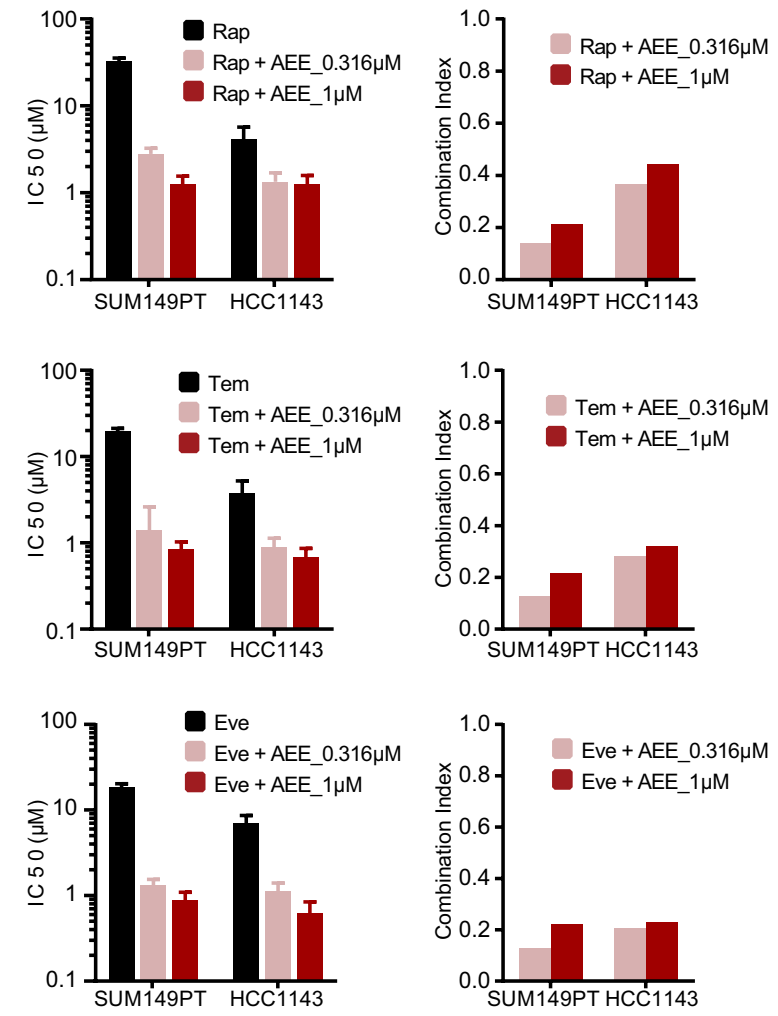

HCC1143
E
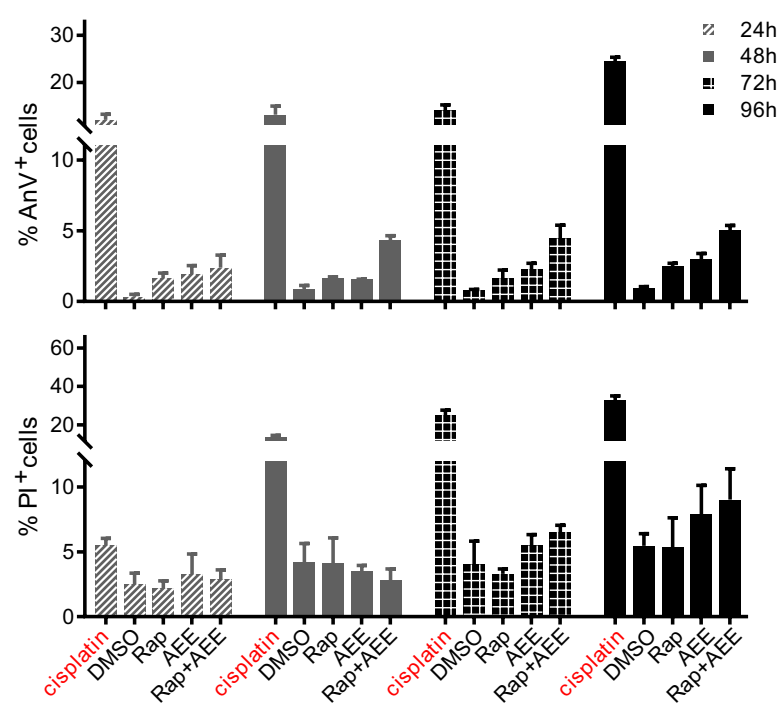

of rapalog and AEE788 in SUM149PT and HCC1143 cells. CI $<1$ indicates synergism. d, e Combinatorial effects of Rap and AEE788 on SUM149PT (left panel, d) and HCC1143 (right panel, e) cell death. Cells were subjected to Annexin V/Propidium Iodide (AnV/ PI) apoptosis assays after treatment for 24, 48, 72, and $96 \mathrm{~h}$. Cisplatin $(100 \mu \mathrm{M})$ was used as positive control. (two-way ANOVA $* p<0.05$, $* * p<0.01, * * * p<0.001)$ 


\section{Co-treatment of rapamycin and AEE788 abolishes $\mathrm{mTOR}$ phosphorylation and sustains downregulation of ERK and AKT signaling in TNBC cells}

mTOR belongs to a complex network of regulatory feedback loops responsible for controlling upstream proliferative signaling pathways. The major upstream signaling in control of mTOR activity involves PI3K/AKT and MAPK/ ERK, the two canonical pathways downstream of RTKs [7]. Resistance to mTOR inhibition in cancer has been linked to activation of upstream PI3K/AKT and MAPK/ERK signaling, following rapalog treatment [15]. Next, we investigated the synergistic effect of AEE788 and rapamycin on PI3K/ AKT and MAPK signaling in TNBC cells. Treatment with AEE788 alone inhibited ERK and AKT phosphorylation in the resistant SUM149PT and HCC1143 cells (Fig. 4a). Single treatment with rapamycin slightly increased p-ERK in SUM149T and p-AKT in HCC1143 cells. The phosphorylation levels of mTOR and the target of mTOR, 4EBP1, were not affected by either AEE788 or rapamycin alone, further indicating the sustained mTOR signaling in the resistant
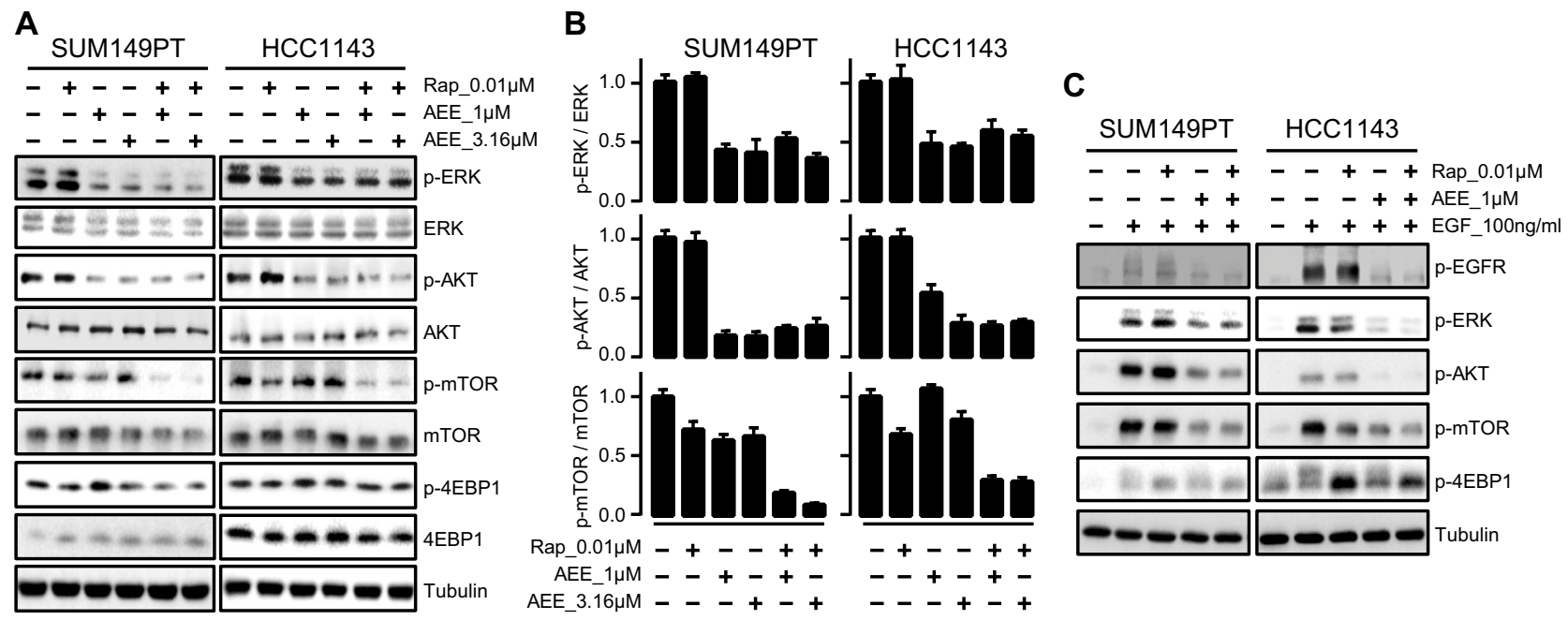

D
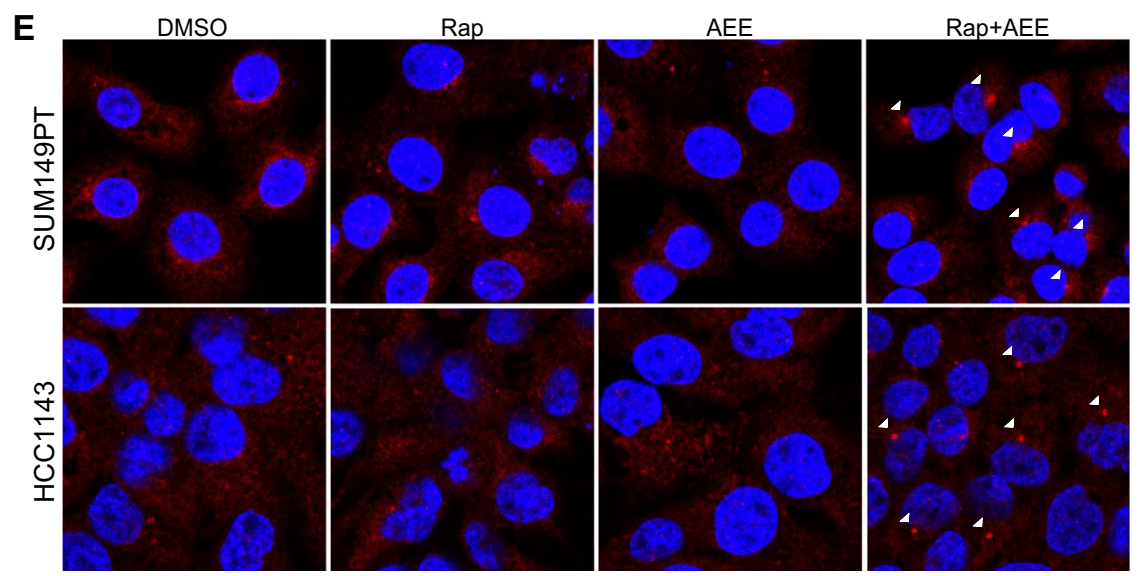

Beclin-1 / Hoechst

Fig. 4 Combinatorial effect of AEE788 and rapamycin on mTOR signaling inhibition in rapalog-resistant TNBC cells. a Effects of AEE788 and Rap co-treatment on mTOR phosphorylation, upstream $\mathrm{AKT}$ and ERK and downstream 4EBP1 signaling in rapalog-resistant SUM149PT and HCC1143 cells. Cells were treated with 1-3.16 $\mu \mathrm{M}$ AEE788 and $0.01 \mu \mathrm{M}$ Rap alone or combined as indicated for $4 \mathrm{~h}$. b Quantification of phosphorylated ERK to total ERK (top row), phosphorylated AKT to total AKT (middle row), and phosphorylated mTOR to total mTOR (bottom row) in SUM149PT and HCC1143 treated with AEE788 and Rap alone or combined as indicated. c Effects of AEE788 and Rap co-treatment on EGF-stimulated sign- aling transduction in SUM149PT and HCC1143 cells. Cells were starved in serum-free medium overnight, pre-treated for $4 \mathrm{~h}$ with AEE788 and Rap alone or combined as indicated, followed by exposure to $100 \mathrm{ng} / \mathrm{ml}$ EGF for $5 \mathrm{~min}$. 4EBP1 can be phosphorylated at several sites, as indicated by multiple bands. The bottom band is the unphosphorylated form of 4EBP1. d Effects of AEE788 and Rap co-treatment for $24 \mathrm{~h}$ on autophagy (LC3B) and ER stress (BiP). e Effects of AEE788 and Rap co-treatment on Beclin-1 accumulation. Cells were subjected to immunofluorescence assay $24 \mathrm{~h}$ post treatment. White arrows indicate the induction of Beclin-1 
TNBC cells. However, co-treatment of AEE788 and rapamycin almost completely abolished mTOR phosphorylation, while ERK and AKT phosphorylation remained inhibited (Fig. 4a, b). The synergistic effect of AEE788 and rapamycin on p-4EBP1 inhibition was marginal (Fig. 4a).

As AEE788 has been described as an EGFR/VEGFR dual RTK inhibitor, we further evaluated the co-treatment effect of AEE788 and rapamycin on EGFR RTK signaling activity in both resistant SUM149PT and HCC1143 cells upon EGF stimulation (Fig. 4c). To reach maximal activation of the EGFR signaling pathway, cells were first serum starved followed by EGF treatment. EGF caused the activation of the EGF receptor as evidenced by increased p-EGFR and downstream p-ERK and p-AKT. AEE788 effectively blocked EGF-stimulated phosphorylation of these components, in both SUM149PT and HCC1143 cells. It has been reported that 4EBP1 has multiple phosphorylation sites and an increase in 4EBP1 phosphorylation is accompanied by a decrease in its electrophoretic mobility [26-28]. EGF also effectively caused enhanced p-mTOR and p-4EBP1. Rapamycin could inhibit 4EBP1 phosphorylation by EGF in both cell lines but with no (SUM149PT) or limited (HCC1143) effects on mTOR activation; and, reversely, AEE788 could inhibit mTOR activation but with marginal effects on 4EBP1 phosphorylation. However, co-treatment with AEE788 and rapamycin particularly could shut down the EGF-mediated phosphorylation of mTOR and 4EBP1 signaling.

Several cellular processes have been linked to the immunogenicity of cell death, including autophagy and ER stress [29-32]. Given that mTOR is a key regulator of autophagy, we then tested the combination effect of autophagy in TNBC cells. Interestingly, the combination sustained the elevated LC3B level induced by monotherapy and demonstrated accumulated Beclin- 1 expression $24 \mathrm{~h}$ post treatment in both SUM149PT and HCC1143 cells (Fig. 4d, e). The combination increased the expression level of BiP, a key regulator of ER stress, $24 \mathrm{~h}$ post treatment in SUM149PT cells, but not so much in HCC1143 cells (Fig. 4d), suggesting the involvement of immunogenic cell death-related events in TNBC cells by the combination treatment.

\section{Silencing of AEE788 targets enhances mTOR inhibition in TNBC cells}

Although AEE788 has been described as an inhibitor targeting multiple RTKs, we wondered whether the effect of AEE788 could be related to unanticipated polypharmacology, thus impacting through additional mechanisms of mTOR signaling. We used a cheminformatics approach to predict candidate alternative kinase targets of AEE788. ChEMBL is an open large-scale bioactivity database that contains comprehensive target inhibition information of thousands of drug-like molecules, including kinase inhibitor activity, allowing well-informed prediction of structurebased alternative kinase target prediction [33, 34]. We firstly performed ligand-based target prediction for AEE788. With $1 \mu \mathrm{M}$ and $10 \mu \mathrm{M}$ activity cutoffs, 9 kinases showed high prediction scores and as such putative targets of AEE788, including RPS6KB1, AKT2, CDK7, EGFR, MAPKAPK2, CLK4, JAK2, AKT3, and VEGFR2 (Fig. 5a). To refine the scale of target list, we selected kinases showing prediction score greater than 0.1 and kinases with an IC50 of AEE788 smaller than $1 \mu \mathrm{M}$ according to the publically available data [35]. As a result, 30 putative kinase targets were selected. To validate the potential contribution of these kinases in the interaction with rapamycin, we performed a targeted rapamycin and siRNA synthetic lethal screen in SUM149PT cells (Fig. 5b). The synthetic lethal screen revealed 13 candidate targets (Fig. 5c). We anticipated that these validated targets would take part in connected signaling networks and, therefore, would all individually impact on the rapamycin sensitivity. Indeed, protein-protein interaction network analysis revealed a close interaction of the various putative kinase targets of AEE788 (Fig. 5d; Suppl. Table S2). Interestingly, the well-known mTOR target RPS6KB1 as well as other RPS6K family members RPS6KA3, RPS6KA6, and RPS6KL1 were mapped in the network, supporting the synergistic drug interaction of AEE788 with rapamycin on mTOR signaling. In addition, ABL2 and PDGFRB were predicted and validated as potential targets involved in rapamycin synergy. Of relevance, rapalog-resistant TNBC cell lines SUM149PT, HCC1143, SUM159PT, and HCC38 poorly responded to inhibitors targeting the verified targets of AEE788, including EGFR, VEGFR, PDGFR, ABL, and S6K (Suppl. Fig. S2). Taken together, the above data suggest that AEE788 synergizes with rapamycin in suppressing TNBC cell proliferation by targeting several EGFR, VEGFR, PDGFR, ABL, and different S6K kinases that are all connected to $\mathrm{mTOR}$ signaling.

\section{AEE788 abolished rapalog-upregulated cyclin D1 expression in TNBC cells}

Finally, we looked into the mechanism how AEE788 and rapalogs impact on cell proliferation. Inhibition of mTOR by rapamycin blocks cell cycle progression, and cell proliferation has been linked to disruption of the cyclin-dependent kinase 4 (CDK4)-cyclin D1 complex [36]. Therefore, we next addressed the role of cyclin D1 in the synergistic effect of AEE788 and rapalogs on proliferation of rapalog-resistant SUM149PT and HCC1143 TNBC cell lines. Unexpectedly, we observed that rapalogs Rap, Tem, and Eve did not suppress but upregulated cyclin D1 protein expression and mRNA levels of CCND1 (the gene encoding cyclin D1) in SUM149PT and HCC1143 TNBC cells after short-term (8 h) and long-term $(24 \mathrm{~h})$ treatments (Fig. $6 \mathrm{a}-\mathrm{c})$. This suggests 
A

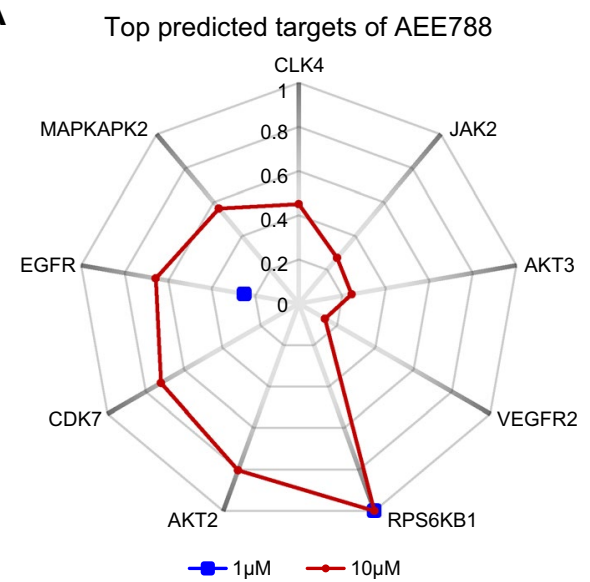

B

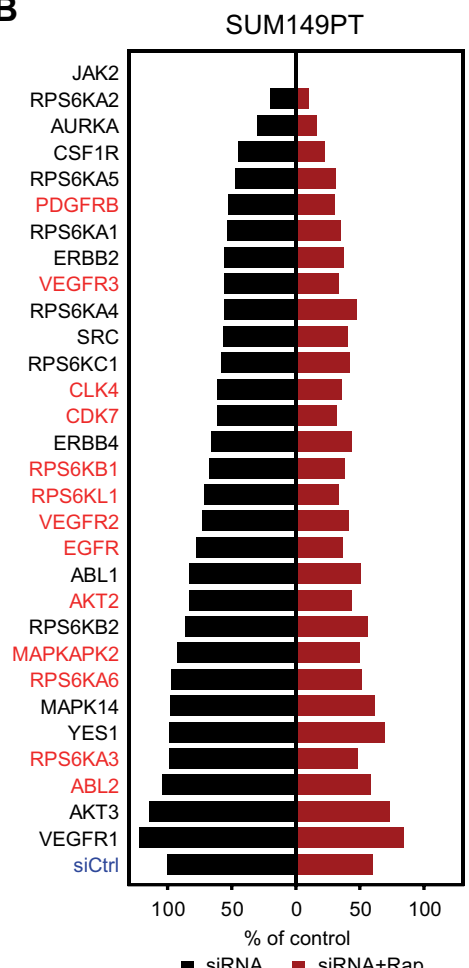

Fig. 5 Validation of AEE788 potential targets synergizing with rapamycin in rapalog-resistant TNBC cells. a Radar chart displaying highly predicted targets of AEE788 in ChEMBL_23 database with $1 \mu \mathrm{M}$ (blue) and $10 \mu \mathrm{M}$ (red) as cutoff. b Screen of AEE788 targets with siRNA alone (siRNA) or in combination with $0.01 \mu \mathrm{M}$ rapamycin (siRNA+Rap) in SUM149PT cells. siCtrl, siRNA control.

a positive-feedback loop activation upon rapalog treatment, thereby counteracting the anti-proliferative effect of rapalogs. Rapalogs slightly increased CDK4 levels, but did not affect cyclin B1 expression in the TNBC cells (Fig. 6a). When co-treated with AEE788, the rapalog-induced cyclin D1 upregulation was blocked at both the mRNA and protein levels (Fig. 6a-c). Moreover, co-treatment of AEE788 and rapalogs led to downregulation of cyclin B1 and CDK4

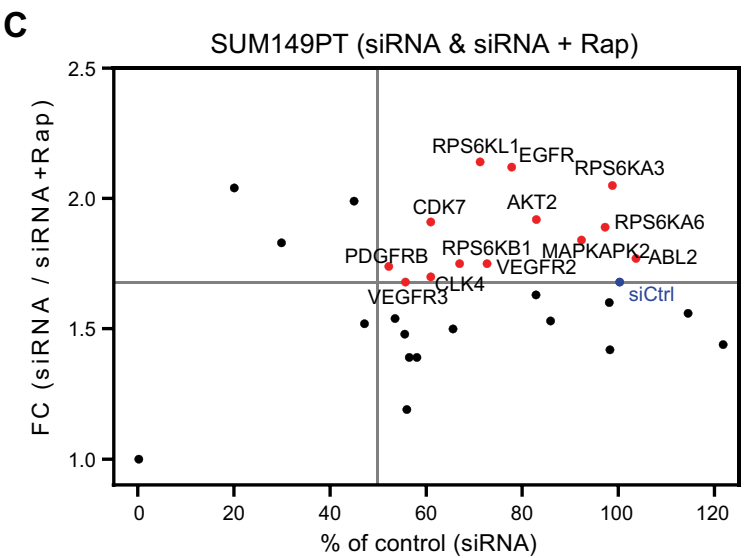

D

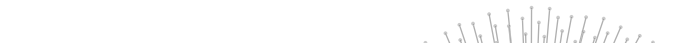


A
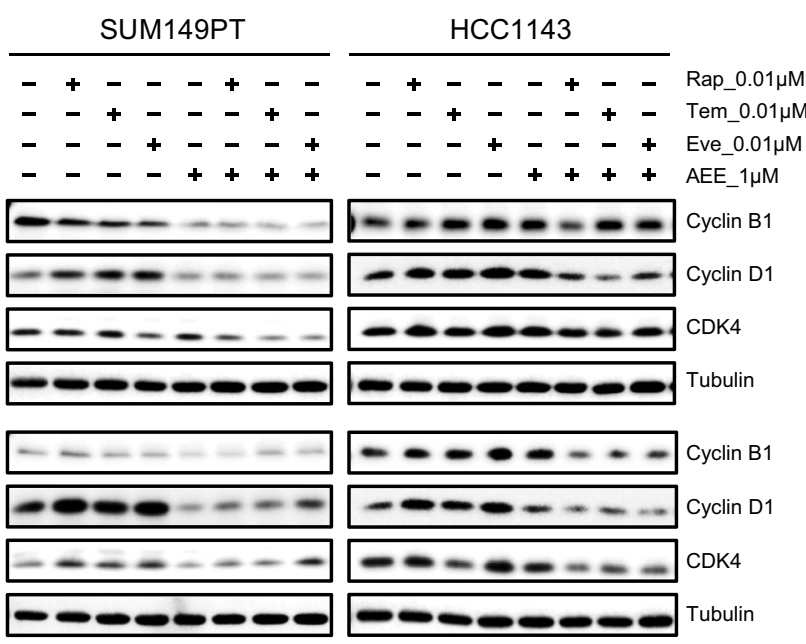

C
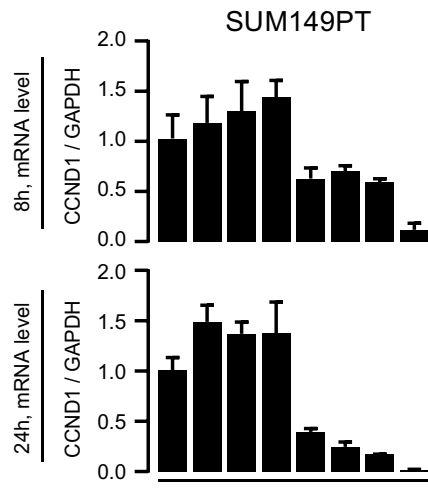

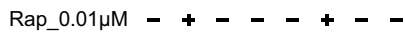

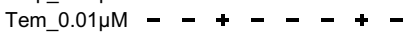

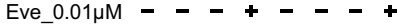

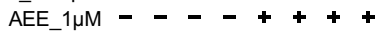

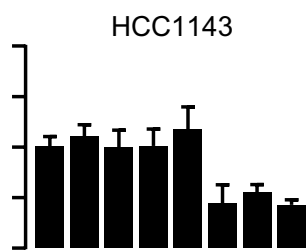

$-+-\quad+\quad+-$

$-+--+$

$--+--+$

$--++++$

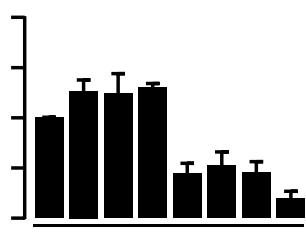

Fig. 6 Co-treatment with AEE788 prevents rapalog-induced cyclin D1 upregulation in resistant TNBC cells. a Combinatorial effects of AEE788 and rapalogs (Rap, Tem, and Eve) on expression of cell cycle regulatory proteins in SUM149PT and HCC1143 cells. Cells were treated with $1 \mu \mathrm{M}$ AEE788 and $0.01 \mu \mathrm{M}$ rapalogs (Rap, Tem, and Eve) alone or combined as indicated, for $8 \mathrm{~h}$ and $24 \mathrm{~h}$, respectively. b Cyclin D1 protein expression levels relative to tubulin in SUM149PT and HCC1143 cells treated with AEE788 and rapa-

significantly enhanced when combined with rapalogs Rap, Tem, or Eve (Fig. 6d). In support of a role of CDK4/cyclin D1 in the resistant phenotype of rapamycin, an enhanced inhibition on proliferation was observed in SUM149PT and HCC 1143 cells when co-treated with rapamycin and selective CDK4/6 inhibitor palbociclib or LY2835219 (Suppl. Fig. S3), albeit not as effective as AEE788.

\section{Discussion}

mTOR acts as a central regulator of multiple signaling networks in control of cell growth, proliferation, and survival $[7,37]$. mTOR signaling is frequently upregulated

B
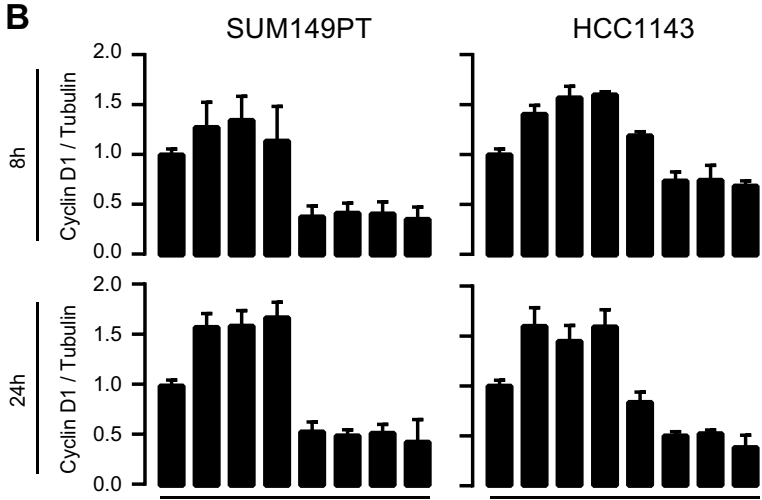

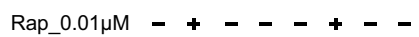

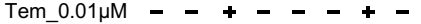

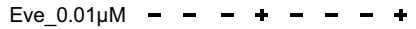

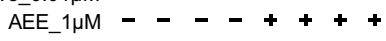

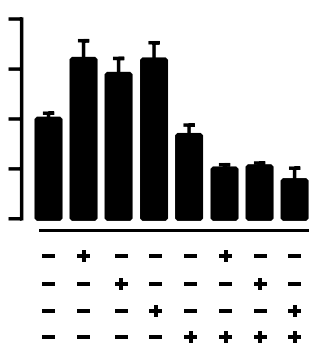

D

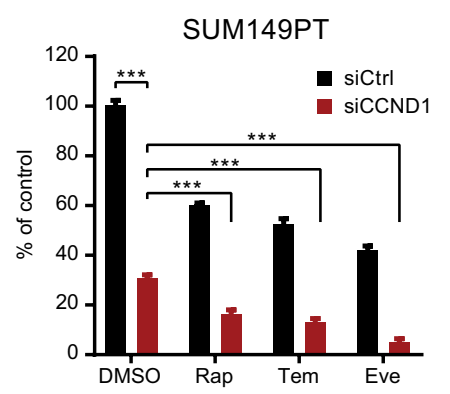

HCC1143

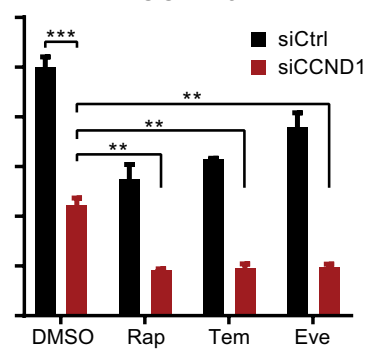

logs alone or combined as indicated. c Cyclin D1 mRNA expression level relative to GAPDH in SUM149PT and HCC1143 cells treated with AEE788 and rapalogs alone or combined as indicated. d Effect of CCND1 siRNA silencing (siCCND1) on proliferation inhibition of SUM149PT and HCC1143 cells treated with $0.01 \mu \mathrm{M}$ rapalogs or DMSO control (two-way ANOVA $* p<0.05$, $* * p<0.01$, $* * * p<0.001)$. siCtrl, siRNA control. Significant effect of rapalogs treatment alone on proliferative inhibition was observed $(* * p<0.01)$

in malignant tumors, including $\mathrm{TNBC}$, highlighting the potential of mTOR kinase targeted therapy in cancer modulation [7-9, 12]. However, patients with TNBC often experience mTOR targeting failure due to acquired resistance and activation of bypass surviving pathways $[13,15$, 38]. Our drug combination screen revealed that co-treatment with AEE788, a multiple RTK-targeted inhibitor, restores the sensitivity of TNBC cells towards the clinically applied mTOR inhibitors (rapamycin, temsirolimus, and everolimus). The effect of AEE788 is likely due to polypharmacology to shut down the crosstalk among receptors as well as mTOR pathway within signaling networks in the resistant scenario. The combination of targeted agents profoundly improves therapeutic efficacy 
and overcomes resistance that might develop under singleagent therapy.

mTOR inhibition can relieve distinct negative feedback loops that normally serve to attenuate upstream RTKs, PI3K, and MAPK signaling, leading to rapalog resistance [39]. As such, mTOR inhibition alone is not sufficient to overcome the entire oncogenic program propagated from the alternate proliferative signaling pathways. By exploiting a high-throughput kinase drug combination screen, our study has identified the effective kinase inhibitor, AEE788, that can block compensatory mechanisms conferring aberrant cell cycle progression upon rapalog treatment. The repression of EGFR/VEGFR- and mTOR-related pathways in concert seemingly reverts processes predominantly responsible for uncontrolled TNBC tumor proliferation. Our results are in line with the above observations that co-inhibition of upstream RTKs (such as EGFR, VEGFR, PDGFR, and IGF1R), PI3K and MAPK signaling transduction, and mTOR signaling elicited enhanced therapeutic efficacy in various cancer types.

Sustained mTOR signaling drives resistance to targeted therapeutics in cancer treatment [38]. In TNBC tumor cells, mTOR signaling is frequently upregulated [40]. We demonstrated that while rapalogs alone were insufficient to inhibit the sustained mTOR signaling in resistant TNBC cells, cotreatment of rapalogs with the multi-targeted RTK inhibitor AEE788 synergistically blocked mTOR phosphorylation in SUM149PT and HCC1143 cells. Interestingly, SUM149PT cells have been characterized for the constitutively activated EGFR via a self-sustaining amphiregulin autocrine loop, and subsequently, altered receptor signaling and gene expression [41, 42]. As a further validation, AEE788 and rapamycin treatment blocked EGF-mediated EGFR downstream signaling in both SUM149PT and HCC1143. We recognize that these experiments were performed under short-term EGF treatment conditions that may not fully reflect the normal TNBC cell signaling. Therefore, we cannot exclude that under more physiological conditions in the tumor microenvironment other distinct RTK signaling cascades are more prominent in the modulation of RTK signaling and cancer progression.

A mechanism of resistance to mTOR inhibition in cancer is the rapalog-mediated activation of upstream PI3K/ AKT and MAPK/ERK signaling [15]. Co-treatment with AEE788 and rapamycin maintained the inhibitory effect on AKT and ERK signaling in TNBC cells. These data suggest that AEE788 and rapamycin synergistically inhibit the sustained mTOR activity in TNBC cells, thus blocking mTOR's potential feedback loop on activation of alternative ERK and AKT proliferative signaling pathways.

Polypharmacology, the action of drugs against multiple targets [17], is commonly observed in drug development including the effective marketed kinase inhibitors [43]. Our
ChEMBL-based cheminformatics analysis demonstrated that AEE788 is a kinase inhibitor that likely targets several kinases; this is consistent with other reports using protein kinase assays [19, 35]. Complementary to a recent kinobeads study on target landscape of clinical kinase drugs [43], our cheminformatics approach presented that the multi-targeted RTK inhibitor AEE788 likely interacts with EGFR, VEGFR, ABL2, PDGFRB, and several mTOR signaling pathway components, including AKT and S6K family members. Subsequent siRNA-based knockdown of these various kinases, e.g., RTKs (EGFR, VEGFR2/3, and PDGFRB), AKTs (AKT2 and AKT3), RPS6Ks (RPS6KA3, RPS6KA6, RPS6KB1, and RPS6KL1), MAPKAPK2, ABL2, and CDK7, sensitized rapalog-resistant TNBC cells to rapamycin. Several reports have demonstrated the synergistic effects of targeting EGFR or MEK on anti-mTOR therapies in TNBC [20, 44-46]. However, our study demonstrated that simultaneous use of EGFRi gefitinib or MEKi PD184352 only exerts additive effects on rapamycin-mediated proliferative inhibition, suggesting that AEE788-rapalog synergy results presumably from multi-targeted kinase inhibition. These data support the anticipated polypharmacology of AEE788 as the mode-of-action of the synergy with rapalogs. Further studies are required to determine the detailed kinome target landscape of AEE788 in TNBC.

mTOR pathway regulates cell growth through its downstream effectors, such as 4EBP1 and RPS6KB1 [7, 37]. Another primary way that mTOR confers its regulatory effects on cell proliferation is to upregulate expression of the cell cycle regulator cyclin D1 [47]. CCND1, the cyclin D1 encoding gene, is frequently amplified in breast cancer, and depletion of cyclin D1 suppresses breast cancer progression $[48,49]$. In response to mTOR inhibition, however, cyclin D1 is elevated by everolimus in various types of cancer $[21,22]$. Consistently, we found that treatment with rapalogs (rapamycin, temsirolimus, and everolimus) commonly upregulated cyclin D1 in rapalog-resistant TNBC cells, indicating an alternative activation of cyclin D1 proliferative signaling pathway after mTOR inhibition. Considering that cyclin D1 was lost in the presence of the AEE788-rapalog combination, AEE788 seems to compensate the undesired effects of rapalog, further highlighting the therapeutic advantage of the drug combination. Interestingly, while we discovered the AEE788-rapamycin interaction through a wider screening effort in TNBC cells, our findings were further supported by the observations on the synergistic effects of AEE788-everolimus combination in prostate, germ, and renal tumor cell lines [19, 21, 22]. Moreover, a xenograftbearing mice study also documented the beneficial action of AEE788-everolimus combination in glioblastoma tumor regression [50]. However, these studies did not further the mode-of-action of AEE788. Since AEE788 is recognized as a multiple targeting kinase inhibitor, their observations 
were limited to EGFR/VEGFR, lacking the notion on other potentially targeted candidate kinases. Our study, for the first time, revealed the synergy on rapalogs treatment in TNBCs and its underlying polypharmacology by utilizing integrated systematic screen and cheminformatics approach. Moreover, either genetic or pharmacological ablation of cyclin D1 significantly enhanced mTOR-inhibition-mediated proliferative inhibition. This is concordant with the recent reports on the synergistic anti-cancer activity of combined CDK4/6 and mTOR targeting [51-53].

In conclusion, our work supports that polypharmacology to target multiple kinase targets in combination with rapalog treatment may offer a distinct combinatorial benefit to TNBC patients that are otherwise resistant to mTORtargeted therapeutics.

Acknowledgements JH was financially supported by the China Scholarship Council.

Author contributions $\mathrm{JH}, \mathrm{YZ}$, and BvdW conceived and designed the experiments. YZ and BvdW supervised the research. JH, RM, VvdN, and $\mathrm{YZ}$ performed the experiments. JM and JF co-supervised the research. GvW performed ligand-based target prediction. JH, YZ, and BvdW wrote the manuscript. All authors read, reviewed, and approved the final manuscript.

Funding This work was supported by the European Research Council Advanced grant Triple-BC (Grant No. 322737) and the Dutch Cancer Society project (Grant No. 2011-5124). GvW was supported by the Dutch Scientific Council (NWO domain AES Veni 14410).

\section{Compliance with ethical standards}

Conflict of interest The authors declare that they have no competing interests.

Ethical approval This article does not contain any studies with human participants or animals performed by any of the authors.

Open Access This article is distributed under the terms of the Creative Commons Attribution 4.0 International License (http://creativecommons.org/licenses/by/4.0/), which permits unrestricted use, distribution, and reproduction in any medium, provided you give appropriate credit to the original author(s) and the source, provide a link to the Creative Commons license, and indicate if changes were made.

\section{References}

1. Podo F, Buydens LM, Degani H, Hilhorst R, Klipp E, Gribbestad IS, Van Huffel S, van Laarhoven HW, Luts J, Monleon D et al (2010) Triple-negative breast cancer: present challenges and new perspectives. Mol Oncol 4(3):209-229

2. Siegel RL, Miller KD, Jemal A (2018) Cancer statistics, 2018. CA Cancer J Clin 68(1):7-30

3. Gluz O, Liedtke C, Gottschalk N, Pusztai L, Nitz U, Harbeck N (2009) Triple-negative breast cancer-current status and future directions. Ann Oncol 20(12):1913-1927
4. Lehmann BD, Bauer JA, Chen X, Sanders ME, Chakravarthy AB, Shyr Y, Pietenpol JA (2011) Identification of human triple-negative breast cancer subtypes and preclinical models for selection of targeted therapies. J Clin Invest 121(7):2750-2767

5. Jhan JR, Andrechek ER (2017) Triple-negative breast cancer and the potential for targeted therapy. Pharmacogenomics 18(17):1595-1609

6. Bianchini G, Balko JM, Mayer IA, Sanders ME, Gianni L (2016) Triple-negative breast cancer: challenges and opportunities of a heterogeneous disease. Nat Rev Clin Oncol 13(11):674-690

7. Saxton RA, Sabatini DM (2017) mTOR signaling in growth, metabolism, and disease. Cell 168(6):960-976

8. Conciatori F, Ciuffreda L, Bazzichetto C, Falcone I, Pilotto S, Bria E, Cognetti F, Milella M (2018) mTOR cross-talk in cancer and potential for combination therapy. Cancers 10(1):23

9. Costa RLB, Han HS, Gradishar WJ (2018) Targeting the PI3K/ AKT/mTOR pathway in triple-negative breast cancer: a review. Breast Cancer Res Treat 169(3):397-406

10. Ueng SH, Chen SC, Chang YS, Hsueh S, Lin YC, Chien HP, Lo YF, Shen SC, Hsueh C (2012) Phosphorylated mTOR expression correlates with poor outcome in early-stage triple negative breast carcinomas. Int J Clin Exp Pathol 5(8):806-813

11. Walsh S, Flanagan L, Quinn C, Evoy D, McDermott EW, Pierce A, Duffy MJ (2012) mTOR in breast cancer: differential expression in triple-negative and non-triple-negative tumors. Breast 21(2):178-182

12. Bahrami A, Khazaei M, Shahidsales S, Hassanian SM, Hasanzadeh M, Maftouh M, Ferns GA, Avan A (2018) The therapeutic potential of PI3K/Akt/mTOR inhibitors in breast cancer: rational and progress. J Cell Biochem 119(1):213-222

13. Carracedo A, Ma L, Teruya-Feldstein J, Rojo F, Salmena L, Alimonti A, Egia A, Sasaki AT, Thomas G, Kozma SC et al (2008) Inhibition of mTORC1 leads to MAPK pathway activation through a PI3K-dependent feedback loop in human cancer. J Clin Invest 118(9):3065-3074

14. Yu YH, Yoon SO, Poulogiannis G, Yang Q, Ma XJM, Villen J, Kubica N, Hoffman GR, Cantley LC, Gygi SP et al (2011) Phosphoproteomic analysis identifies Grb10 as an mTORC1 substrate that negatively regulates insulin signaling. Science 332(6035):1322-1326

15. Faes S, Demartines N, Dormond O (2017) Resistance to mTORC1 inhibitors in cancer therapy: from kinase mutations to intratumoral heterogeneity of kinase activity. Oxid Med Cell Longev 2017:1726078

16. Chalakur-Ramireddy NKR, Pakala SB (2018) Combined drug therapeutic strategies for the effective treatment of triple negative breast cancer. Biosci Rep 38(1):BSR20171357

17. Ma XD, Lv XQ, Zhang JK (2018) Exploiting polypharmacology for improving therapeutic outcome of kinase inhibitors (KIs): an update of recent medicinal chemistry efforts. Eur J Med Chem 143:449-463

18. Sticz T, Molnar A, Danko T, Hujber Z, Petovari G, Nagy N, Vegso G, Kopper L, Sebestyen A (2018) The effects of different mTOR inhibitors in EGFR inhibitor resistant colon carcinoma cells. Pathol Oncol Res. https://doi.org/10.1007/s12253-018-0434-4

19. Schaffrath J, Schmoll HJ, Voigt W, Muller LP, Muller-Tidow C, Mueller T (2017) Efficacy of targeted drugs in germ cell cancer cell lines with differential cisplatin sensitivity. PLoS ONE 12(6): 0178930

20. Liu T, Yacoub R, Taliaferro-Smith LD, Sun SY, Graham TR, Dolan R, Lobo C, Tighiouart M, Yang L, Adams A et al (2011) Combinatorial effects of lapatinib and rapamycin in triple-negative breast cancer cells. Mol Cancer Ther 10(8):1460-1469

21. Wedel S, Hudak L, Seibel JM, Juengel E, Tsaur I, Haferkamp A, Blaheta RA (2011) Combined targeting of the VEGFr/EGFr and the mammalian target of rapamycin (mTOR) signaling pathway 
delays cell cycle progression and alters adhesion behavior of prostate carcinoma cells. Cancer Lett 301(1):17-28

22. Juengel E, Engler J, Natsheh I, Jones J, Mickuckyte A, Hudak L, Jonas D, Blaheta RA (2009) Combining the receptor tyrosine kinase inhibitor AEE788 and the mammalian target of rapamycin (mTOR) inhibitor RAD001 strongly inhibits adhesion and growth of renal cell carcinoma cells. BMC Cancer 9:161

23. Zhang Y, Moerkens M, Ramaiahgari S, de Bont H, Price L, Meerman J, van de Water B (2011) Elevated insulin-like growth factor 1 receptor signaling induces antiestrogen resistance through the MAPK/ERK and PI3K/Akt signaling routes. Breast Cancer Res 13(3):R52

24. Ballou LM, Lin RZ (2008) Rapamycin and mTOR kinase inhibitors. J Chem Biol 1(1-4):27-36

25. Chiang GG, Abraham RT (2005) Phosphorylation of mammalian target of rapamycin (mTOR) at ser-2448 is mediated by p70S6 kinase. J Biol Chem 280(27):25485-25490

26. Musa J, Orth MF, Dallmayer M, Baldauf M, Pardo C, Rotblat B, Kirchner T, Leprivier G, Grunewald TG (2016) Eukaryotic initiation factor 4E-binding protein 1 (4E-BP1): a master regulator of mRNA translation involved in tumorigenesis. Oncogene 35(36):4675-4688

27. Feun L, You M, Wu CJ, Kuo MT, Wangpaichitr M, Spector S, Savaraj N (2008) Arginine deprivation as a targeted therapy for cancer. Curr Pharm Des 14(11):1049-1057

28. Gingras AC, Gygi SP, Raught B, Polakiewicz RD, Abraham RT, Hoekstra MF, Aebersold R, Sonenberg N (1999) Regulation of 4E-BP1 phosphorylation: a novel two-step mechanism. Genes Dev 13(11):1422-1437

29. Galluzzi L, Buque A, Kepp O, Zitvogel L, Kroemer G (2017) Immunogenic cell death in cancer and infectious disease. Nat Rev Immunol 17(2):97-111

30. Kepp O, Senovilla L, Vitale I, Vacchelli E, Adjemian S, Agostinis P, Apetoh L, Aranda F, Barnaba V, Bloy N et al (2014) Consensus guidelines for the detection of immunogenic cell death. Oncoimmunology 3(9):e955691

31. Rufo N, Garg AD, Agostinis P (2017) The unfolded protein response in immunogenic cell death and cancer immunotherapy. Trends Cancer 3(9):643-658

32. Kepp O, Semeraro M, Bravo-San Pedro JM, Bloy N, Buque A, Huang X, Zhou H, Senovilla L, Kroemer G, Galluzzi L (2015) eIF2alpha phosphorylation as a biomarker of immunogenic cell death. Semin Cancer Biol 33:86-92

33. Schenone M, Dancik V, Wagner BK, Clemons PA (2013) Target identification and mechanism of action in chemical biology and drug discovery. Nat Chem Biol 9(4):232-240

34. Papadatos G, Gaulton A, Hersey A, Overington JP (2015) Activity, assay and target data curation and quality in the ChEMBL database. J Comput Aided Mol Des 29(9):885-896

35. Traxler P, Allegrini PR, Brandt R, Brueggen J, Cozens R, Fabbro D, Grosios K, Lane HA, McSheehy P, Mestan J et al (2004) AEE788: a dual family epidermal growth factor receptor/ErbB2 and vascular endothelial growth factor receptor tyrosine kinase inhibitor with antitumor and antiangiogenic activity. Cancer Res 64(14):4931-4941

36. Jiang BH, Liu LZ (2008) Role of mTOR in anticancer drug resistance: perspectives for improved drug treatment. Drug Resist Updates 11(3):63-76

37. Hare SH, Harvey AJ (2017) mTOR function and therapeutic targeting in breast cancer. Am J Cancer Res 7(3):383-404

38. Ilagan E, Manning BD (2016) Emerging role of mTOR in the response to cancer therapeutics. Trends Cancer 2(5):241-251

39. Efeyan A, Sabatini DM (2010) mTOR and cancer: many loops in one pathway. Curr Opin Cell Biol 22(2):169-176

40. She QB, Gruvberger-Saal SK, Maurer M, Chen Y, Jumppanen M, Su T, Dendy M, Lau YK, Memeo L, Horlings HM et al (2016)
Integrated molecular pathway analysis informs a synergistic combination therapy targeting PTEN/PI3K and EGFR pathways for basal-like breast cancer. BMC Cancer 16:587

41. Willmarth NE, Ethier SP (2006) Autocrine and juxtacrine effects of amphiregulin on the proliferative, invasive, and migratory properties of normal and neoplastic human mammary epithelial cells. J Biol Chem 281(49):37728-37737

42. Kappler CS, Guest ST, Irish JC, Garrett-Mayer E, Kratche Z, Wilson RC, Ethier SP (2015) Oncogenic signaling in amphiregulin and EGFR-expressing PTEN-null human breast cancer. Mol Oncol 9(2):527-543

43. Klaeger S, Heinzlmeir S, Wilhelm M, Polzer H, Vick B, Koenig PA, Reinecke M, Ruprecht B, Petzoldt S, Meng C et al (2017) The target landscape of clinical kinase drugs. Science 358(6367):eaan4368

44. Fujishita T, Kojima Y, Kajino-Sakamoto R, Taketo MM, Aoki M (2017) Tumor microenvironment confers mTOR inhibitor resistance in invasive intestinal adenocarcinoma. Oncogene 36(46):6480-6489

45. Madden JM, Mueller KL, Bollig-Fischer A, Stemmer P, Mattingly RR, Boerner JL (2014) Abrogating phosphorylation of eIF4B is required for EGFR and mTOR inhibitor synergy in triple-negative breast cancer. Breast Cancer Res Treat 147(2):283-293

46. You KS, Yi YW, Kwak SJ, Seong YS (2018) Inhibition of RPTOR overcomes resistance to EGFR inhibition in triple-negative breast cancer cells. Int J Oncol 52(3):828-840

47. Advani SH (2010) Targeting mTOR pathway: a new concept in cancer therapy. Indian J Med Paediatr Oncol 31(4):132-136

48. Laphanuwat P, Likasitwatanakul P, Sittithumcharee G, Thaphaengphan A, Chomanee N, Suppramote O, Ketaroonrut N, Charngkaew K, Lam EW, Okada S et al (2018) Cyclin D1 depletion interferes with oxidative balance and promotes cancer cell senescence. J Cell Sci 131(12):jcs214726

49. Sanchez-Vega F, Mina M, Armenia J, Chatila WK, Luna A, La KC, Dimitriadoy S, Liu DL, Kantheti HS, Saghafinia S et al (2018) Oncogenic signaling pathways in the cancer genome atlas. Cell 173(2):321-337

50. Goudar RK, Shi Q, Hjelmeland MD, Keir ST, McLendon RE, Wikstrand CJ, Reese ED, Conrad CA, Traxler P, Lane HA et al (2005) Combination therapy of inhibitors of epidermal growth factor receptor/vascular endothelial growth factor receptor 2 (AEE788) and the mammalian target of rapamycin (RAD001) offers improved glioblastoma tumor growth inhibition. Mol Cancer Ther 4(1): 101-112

51. Cretella D, Ravelli A, Fumarola C, La Monica S, Digiacomo G, Cavazzoni A, Alfieri R, Biondi A, Generali D, Bonelli $\mathrm{M}$ et al (2018) The anti-tumor efficacy of CDK4/6 inhibition is enhanced by the combination with PI3K/AKT/mTOR inhibitors through impairment of glucose metabolism in TNBC cells. J Exp Clin Cancer Res 37(1):72

52. Yamamoto T, Kanaya N, Somlo G, Chen S (2019) Synergistic anti-cancer activity of CDK4/6 inhibitor palbociclib and dual mTOR kinase inhibitor MLN0128 in pRb-expressing ER-negative breast cancer. Breast Cancer Res Treat 174(3):615-625

53. Chen L, Yang G, Dong H (2019) Everolimus reverses palbociclib resistance in ER+ human breast cancer cells by inhibiting phosphatidylinositol 3-kinase(PI3K)/Akt/mammalian target of rapamycin (mTOR) pathway. Med Sci Monit 25:77-86

Publisher's Note Springer Nature remains neutral with regard to jurisdictional claims in published maps and institutional affiliations. 